\title{
ON ANALYTIC FUNCTIONS WITH BOUNDED CHARACTERISTIC
}

\section{HANS FRIED ${ }^{1}$}

A function $f\left(r e^{i \phi}\right)$, regular within the unit circle, is called a function with bounded characteristic if

$$
\lim _{r \rightarrow 1} \int_{0}^{2 \pi} \log ^{+}\left|f\left(r e^{i \phi}\right)\right| d \phi
$$

is bounded, where $\log ^{+}\left|f\left(r e^{i \phi}\right)\right|=\max \left(\log \left|f\left(r e^{i \phi}\right)\right|, 0\right)$. If $f(z)$ is a function with bounded characteristic, then

$$
\lim _{r \rightarrow 1} f\left(r e^{i \phi}\right)=f\left(e^{i \phi}\right)
$$

exists almost everywhere [1].2

In the first part of this paper we prove the following:

Theorem I. Let $\left\{f_{n}(z)\right\}(n=1,2,3, \cdots)$ and $f(z)$ be functions with bounded characteristics, let

$$
\begin{aligned}
& \log A_{n}=\lim _{r \rightarrow 1} \int_{0}^{2 \pi} \log ^{+}\left|f_{n}\left(r e^{i \phi}\right)\right| d \phi, \\
& \log A=\lim _{r \rightarrow 1} \int_{0}^{2 \pi} \log ^{+}\left|f\left(r e^{i \phi}\right)\right| d \phi,
\end{aligned}
$$

and

(2) $\left|f\left(e^{i \phi}\right)-f_{n}\left(e^{i \phi}\right)\right|<m_{n}$, for $\phi \in E_{n}$, and let $\mu_{n}$ be the measure of $E_{n}$.

If

$$
\lim _{n \rightarrow \infty} m_{n}^{\mu_{n}}=0
$$

and for every positive $\sigma$ there exists a positive integer $n_{\sigma}$ such that

$$
A_{n}<m_{n}^{-\sigma \mu_{n}} \text { for } n>n_{\sigma},
$$

then the sequence $\left\{f_{n}(z)\right\}$ tends uniformly to $f(z)$ in any closed domain interior to the unit circle.

Received by the editors January $8,1946$.

1 Deceased December 23, 1945.

${ }^{2}$ Numbers in brackets refer to the Bibliography at the end of the paper. 
Special cases of this theorem are the following:

A. If the numbers $A_{n}$ are bounded, we need only the condition (3), as (4) follows from (3) in this case.

B. If the numbers $A_{n}$ are bounded, $E_{1}=E_{2}=\cdots=E_{n}=\cdots$ and $\mu_{1}>0$, we need only the condition $\lim _{n \rightarrow \infty} m_{n}=0$ instead of (3) and (4). This case was proved by Ostrowski [1].

C. If $E_{1}=E_{2}=\cdots=E_{n}=\cdots$ and $\mu_{1}>0$, we can replace (3) and (4) by the following two conditions: $\lim _{n \rightarrow \infty} m_{n}=0$, and for every positive $\sigma$ there exists a positive integer $n_{\sigma}$ such that $A_{n}<m_{n}^{-\sigma}$ for $n>n_{\sigma}$. This case was proved by Milloux [2] under the less general assumptions that the functions $f_{n}(z)$ and $f(z)$ are bounded, and that $E_{1}=E_{2}=\cdots=E_{n}=\cdots$ is an arc $(\alpha, \beta)$ of the unit circle on which the functions $f_{n}(z)$ and $f(z)$ are continuous.

In the second part of this paper we prove the following theorem.

THEOREM II. Let

$$
f(z)=\sum_{k=1}^{\infty} c_{k} z^{n_{k}}
$$

be a function with bounded characteristic and let

$$
\frac{n_{k+1}}{n_{k}} \geqq q>1 \quad(k=1,2, \cdots) ;
$$

then

$$
\sum_{k=1}^{\infty}\left|c_{k}\right|^{2}
$$

converges.

This theorem generalizes a theorem of R. Paley [3], which proved that if $f(z)$ has an expansion (5) where the $n_{k}$ satisfy (6) and

$$
\lim _{r \rightarrow 1} \int_{0}^{2 \pi}\left|f\left(r e^{i \phi}\right)\right| d \phi
$$

is bounded, then the series (7) converges. From $\log ^{+}|f(z)|<|f(z)|$ it follows that each function which satisfies (8) is of bounded characteristic. Therefore, Paley's theorem is a consequence of Theorem II. We shall show that Theorem II gives a negative answer to the following question asked by Bloch [4]: If $f(z)$ is a function with bounded characteristic, must the derivative of $f(z)$ have the same property?

1. Proof of Theorem I. We put $\phi_{n}(z)=f(z)-f_{n}(z)$ and $\phi_{n}\left(e^{i \phi}\right)$ $=f\left(e^{i \phi}\right)-f_{n}\left(e^{i \phi}\right)$. We have 


$$
\begin{aligned}
\log ^{+}\left|\phi_{n}(z)\right| & =\log ^{+}\left|f(z)-f_{n}(z)\right| \leqq \log ^{+}\left(|f(z)|+\left|f_{n}(z)\right|\right) \\
& \leqq \log ^{+}|f(z)|+\log ^{+}\left|f_{n}(z)\right|+\log 2 .
\end{aligned}
$$

Let $\left\{r_{k}\right\}$ be an increasing sequence of positive numbers with

$$
\lim _{x \rightarrow \infty} r_{k}=1 \text {. }
$$

By Jensen's formula we have, for $r<r_{k}$,

$$
\begin{aligned}
\log \left|\phi_{n}\left(r e^{i \phi}\right)\right|+\sum_{\mu=1}^{m} \log & \left|\frac{\stackrel{2}{k}_{k}-\bar{a}_{\mu} r e^{i \phi}}{r_{k}\left(r e^{i \phi}-a_{\mu}\right)}\right| \\
& =\frac{1}{2 \pi} \int_{0}^{2 \pi} \frac{\left(r_{k}^{2}-r^{2}\right) \log \left|\phi_{n}\left(r_{k} e^{i \theta}\right)\right|}{r_{k}^{2}-2 r_{k} r \cos (\phi-\theta)+r^{2}} d \theta
\end{aligned}
$$

where $a_{1}, a_{2}, \cdots, a_{m}$ are the zeros of $\phi_{n}(z)$ within the circle of radius $r_{k}$. As the second term on the left side of (10) is positive we have

$$
2 \pi \log \left|\phi_{n}\left(r e^{i \phi}\right)\right| \leqq \int_{0}^{2 \pi} \frac{\left(r_{k}^{2}-r^{2}\right) \log \left|\phi_{n}\left(r_{k} e^{i \theta}\right)\right| d \theta}{r_{k}^{2}-2 r_{k} r \cos (\phi-\theta)+r^{2}} .
$$

As $\lim _{k \rightarrow \infty} \phi_{n}\left(r_{k} e^{i \phi}\right)=\phi_{n}\left(e^{i \phi}\right)$ for $\phi \in E_{n}$, there exists by Egoroff's theorem, for every positive $\delta_{1}$, a set $E_{n}{ }^{\prime}<E_{n}(n=1,2, \cdots)$ such that

$$
\mu\left(E_{n}^{\prime}\right)>\mu_{n}-\delta_{1},
$$

and

$$
\lim _{\boldsymbol{x} \rightarrow \infty} \phi_{n}\left(r_{k} e^{i \phi}\right)=\phi_{n}\left(e^{i \phi}\right)
$$

uniformly in $E_{n}^{\prime}$.

Because of (3) we can assume $m_{n}<1(n=1,2, \cdots)$; hence it follows from (2) and (13), that

$$
\left|\phi_{n}\left(r_{k} e^{i \phi}\right)\right|<m_{n}+\delta_{2}<1 \text {, }
$$

for $\phi \in E_{n}^{\prime}$ and for sufficiently large $k$, where $\delta_{2}$ is arbitrarily small. We denote by $E_{n}^{\prime \prime}$ the complement of $E_{n}^{\prime}$ with respect to $(0,2 \pi)$. Then (11) takes the form

$$
\begin{aligned}
2 \pi \log \left|\phi_{n}\left(r e^{i \phi}\right)\right| \leqq & \int_{E_{n}^{\prime \prime}} \frac{\left(r_{k}^{2}-r^{2}\right) \log { }^{+}\left|\phi_{n}\left(r_{k} e^{i \theta}\right)\right| d \theta}{r_{k}^{2}-2 r_{k} r \cos (\phi-\theta)+r^{2}} \\
& +\int_{E_{n}^{\prime}} \frac{\left(r_{k}^{2}-r^{2}\right) \log \left|\phi_{n}\left(r_{k} e^{i \theta}\right)\right| d \theta}{r_{k}^{2}-2 r_{k} r \cos (\phi-\theta)+r^{2}}
\end{aligned}
$$


As the first integral on the right side is positive and the second negative, we have, because of (1), (9) and (14),

$$
\begin{aligned}
2 \pi \log \left|\phi_{n}\left(r e^{i \phi}\right)\right| \leqq & \frac{r_{k}+r}{r_{k}-r} \int_{E_{n}^{\prime \prime}} \log +\left|\phi_{n}\left(r e^{i \theta}\right)\right| d \theta \\
& +\frac{r_{k}-r}{r_{k}+r} \int_{E_{n}^{\prime}} \log \left|\phi_{n}\left(r e^{i \theta}\right)\right| d \theta \\
\leqq & \frac{r_{k}+r}{r_{k}-r} \log 2 A A_{n} \\
& +\frac{r_{k}-r}{r_{k}+r}\left(\mu_{n}-\delta_{1}\right) \log \left(m_{n}+\delta_{2}\right),
\end{aligned}
$$

for sufficiently large $k$. We get, therefore, for $\lim _{k \rightarrow \infty} r_{k}=1$, as $\delta_{1}$ and $\delta_{2}$ are arbitrarily small,

$$
2 \pi \log \left|\phi_{n}\left(r e^{i \phi}\right)\right| \leqq \frac{1+r}{1-r} \log 2 A A_{n}+\frac{1-r}{1+r} \mu_{n} \log m_{n}
$$

or

$$
\left|\phi_{n}\left(r e^{i \phi}\right)\right|=\left(2 A A_{n}\right)^{(1+r) / 2 \pi(1-r)} m_{n}{ }_{n}{ }^{\mu_{n}(1-r) / 2 \pi(1+r)} .
$$

Let $B$ be any closed domain within the unit circle. There exists an $r^{\prime}<1$, such that the interior of the circle with radius $r^{\prime}$ contains $B$. Now we choose

$$
\sigma<\left(\frac{1-r^{\prime}}{1+r^{\prime}}\right)^{2}
$$

If we put $A^{\prime}=\max (A, 1)$ and $A_{n}^{\prime}=\max \left(A_{n}, 1\right)$ we get, for $r e^{i \phi} \in B$, by use of (4),

$$
\left|\phi_{n}\left(r e^{i \phi}\right)\right| \leqq\left(2 A^{\prime}\right)^{\left(1+r^{\prime}\right) / 2 \pi\left(1-r^{\prime}\right)} m_{n}^{\left(\mu_{n} / 2 \pi\right)\left(\left(1-r^{\prime}\right) /\left(1+r^{\prime}\right)-\sigma\left(1+r^{\prime}\right) /\left(1-r^{\prime}\right)\right)}
$$

for sufficiently large $n$.

As, because of $(16),\left(1-r^{\prime}\right) /\left(1+r^{\prime}\right)-\sigma\left(1+r^{\prime}\right) /\left(1-r^{\prime}\right)>0$ and since, by assumption, $\lim _{n \rightarrow \infty} m_{n}{ }^{\mu_{n}}=0$, we have proved the theorem.

2. Proof of Theorem II. For the proof of Theorem II we need the following two theorems, the first due to Hardy and Littlewood [5], the second to Zygmund [6].

Theorem A. If $f(z)=\sum_{k=1}^{\infty} c_{k} z^{n_{k}}$ and $n_{k+1} / n_{k} \geqq q>1(k=1,2, \cdots)$, then the existence of $\lim _{r \rightarrow 1} f\left(r e^{i \phi_{0}}\right)=f\left(e^{i \phi_{0}}\right)$ implies the convergence of $\sum_{k=1}^{\infty} c_{k} e^{i n_{k} \phi 0}$. 
THEOREM B. If the trigonometric series

$$
\sum_{k=1}^{\infty}\left(a_{k} \cos n_{k} \theta+b_{k} \sin n_{k} \theta\right) \quad\left(n_{k}+1 / n_{k} \geqq q>1\right)
$$

converges in a set of positive measure, then the series

$$
\sum_{k=1}^{\infty}\left(a_{k}^{2}+b_{k}^{2}\right)
$$

converges.

As $f(z)$ is a function with bounded characteristic

$$
\lim _{r \rightarrow 1} f\left(r e^{i \phi}\right)=f\left(e^{i \phi}\right)
$$

exists almost everywhere. If we put $c_{k}=a_{k}-i b_{k}$ then by Theorem $\mathrm{A}$ the series

$$
\begin{aligned}
\sum_{k=1}^{\infty}\left(a_{k}-i b_{k}\right)\left(\cos n_{k} \phi+i \sin n_{k} \phi\right)= & \sum_{k=1}^{\infty}\left(a_{k} \cos n_{k} \phi+b_{k} \sin n_{k} \phi\right) \\
& +i \sum_{k=1}^{\infty}\left(a_{k} \sin n_{k} \phi-b_{k} \cos n_{k} \phi\right)
\end{aligned}
$$

converges almost everywhere. Therefore, by Theorem B, the series

$$
\sum_{k=1}^{\infty} a_{k}^{2}+b_{k}^{2}=\sum_{k=1}^{\infty}\left|c_{k}\right|^{2}
$$

converges.

Now we shall show that there exist bounded functions, whose derivatives are functions whose characteristics are not bounded.

The radius of convergence of the power series

$$
f(z)=\sum_{k=1}^{\infty} \frac{z^{n_{k}}}{n_{k}} \quad\left(\frac{n_{k+1}}{n_{k}} \geqq q>1\right)
$$

is equal to 1 and the function $f(z)$ is bounded within the unit circle. The derivative

$$
f^{\prime}(z)=\sum_{k=1}^{\infty} z^{n_{k-1}}
$$

is, by Theorem II, a function whose characteristic is not bounded. 


\section{BiBLIOGRAPHY}

1. A. Ostrowski, Über die Bedeutung der Jensenschen Formal fïr einige Pragen der komplexen Functionentheorie, Acta Univ. Szeged. vol. 1 (1923) pp. 80-87.

2. H. Milloux, Le thêrème de M. Picard, suites de fonctions holomorphes, fonctions méromorphes et fonctions entières, J. Math. Pures Appl. (9) vol. 3 (1924) pp. 345-401.

3. R. E. A. C. Paley, On the lacunary coefficients of power series, Ann. of Math. vol. 34 (1933) pp. 615-616.

4. R. Nevanlinna, Le theorème de Picard-Borel et la theorie des fonctions meromorphes, Paris, 1929, pp. 133 and 138.

5. G. H. Hardy and J. E. Littlewood, $A$ further note on the converse of Abel's theorem, Proc. London Math. Soc. (2) vol. 25 (1926) pp. 219-236.

6. A. Zygmund, On the convergence of lacunary trigonometric series, Fund. Math. vol. 16 (1930) pp. 90-107.

Swarthmore College

\section{ON THE $(C, 1)$ SUMMABILITY OF CERTAIN RANDOM SEQUENCES}

\section{HERBERT ROBBINS}

It is known [1] $]^{1}$ that if a sequence $\left\{a_{n}\right\}(n=1,2, \cdots)$ of real numbers is summable $(C, 1)$ to a value $\alpha$, and if $\sum a_{n}^{2} / n^{2}<\infty$, then almost all the subsequences of $\left\{a_{n}\right\}$ are summable $(C, 1)$ to $\alpha$. It will be shown that this statement continues to hold if "almost all" is replaced by "with probability 1 " and "subsequences" by the more general term "product sequences," the meaning of which will be defined in the next paragraph. The only analytic tool used is the strong law of large numbers [2]: if $\left\{y_{n}\right\}$ is a sequence of independent random variables with expected values $E\left(y_{n}\right)=0$ and $E\left(y_{n}{ }^{2}\right)=b_{n}{ }^{2}$, for which $\sum b_{n}{ }^{2} / n^{2}<\infty$, then with probability 1 the sequence $\left\{y_{n}\right\}$ is summable $(C, 1)$ to the value 0 .

DEFinition. Let $\left\{a_{n}\right\}$ be a sequence of constants and let $\left\{x_{n}\right\}$ be a sequence of random variables such that the values of each $x_{n}$ are non-negative integers. For every $n$ let $k(n)$ be the least positive integer $m$ such that

$$
\sum_{1}^{m} x_{i} \geqq n
$$

Presented to the Society, September 17, 1945; received by the editors January 23, 1946.

1 Numbers in brackets refer to references listed at end of paper. 